\title{
On the nature of X-ray flashes
}

\author{
C. Barraud ${ }^{1}$, F. Daigne ${ }^{2,3}$, R. Mochkovitch ${ }^{2}$, and J. L. Atteia ${ }^{1}$ \\ ${ }^{1}$ Laboratoire d'Astrophysique de Toulouse-Tarbes, OMP, 31400 Toulouse, France \\ 2 Institut d'Astrophysique de Paris, 98 bis boulevard Arago, 75014 Paris, France \\ e-mail: mochko@iap.fr \\ ${ }^{3}$ Université Pierre et Marie Curie - Paris VI, 4 Place Jussieu, 75005 Paris, France
}

Received 1 July 2004 / Accepted 8 April 2005

\begin{abstract}
We discuss the origin of X-Ray Flashes (XRFs), a recently discovered class of Gamma-Ray Bursts (GRBs). Using a simplified model for internal shocks we check if XRFs can be intrinsically soft due to some specific values of the parameters describing the relativistic outflow emerging from the central engine. We generate a large number of synthetic events and find that XRFs are obtained when the contrast $\Gamma_{\max } / \Gamma_{\min }$ of the Lorentz factor distribution is small while the average Lorentz factor $\bar{\Gamma}$ is large. A few XRFs may be GRBs at large redshifts but we exclude this possibility for the bulk of the population. If outflows with a small contrast are commonly produced, even a large population of XRFs could be explained. If conversely the Lorentz factor distribution within the wind is broad, one should then rely on extrinsic causes, such as viewing angle effects or high redshift.
\end{abstract}

Key words. gamma rays: bursts - shock waves - radiation mechanisms: non thermal

\section{Introduction}

An intriguing discovery in recent years is the existence of a population of soft gamma-ray bursts (GRBs) with little or no emission above $50 \mathrm{keV}$ (Heise et al. 2001; Kippen et al. 2001; Barraud et al. 2003). These events, which have been called X-Ray Flashes (XRFs), share a number of characteristics with the classical GRBs (long duration, non-thermal spectra ...), and there is now general consensus on the fact that XRFs represent an extension at low energies of the GRB population. In this context it is natural to verify whether the models developed to explain the prompt emission of GRBs can also explain a population of soft bursts like the XRFs. This is a complex issue because XRFs can be explained either by extrinsic factors (e.g. viewing angle, redshift) or intrinsic factors (e.g. Lorentz factor, energy deposition...). An overview of the factors that could give rise to soft GRBs appeared in Zhang \& Meszaros (2002). Recently several authors have discussed in detail the effects of the viewing angle on the softness of GRBs (e.g. Yamazaki et al. 2002; Zhang et al. 2004; Lamb et al. 2004).

In this paper we concentrate on the impact of intrinsic parameters and we specifically address the following question: can the internal shocks model, which successfully explains many properties of the GRB prompt emission, also explain XRFs without calling upon a particular set of extrinsic factors? Our work is based on an analytical model that captures the essential physics of internal shocks. We demonstrate that internal shocks can produce XRFs quite naturally and we discuss the conditions required for this to happen.
The paper is organized as follows. Section 2 summarizes our current knowledge of XRFs. Section 3 introduces our analytical model of internal shocks. Section 4 presents the results of the simulation of a large number of GRBs and discusses the conditions required to produce XRFs. Section 5 summarizes our results and presents our conclusions.

\section{Observational properties of XRFs}

\subsection{Gamma-ray bursts and X-ray flashes}

In 2001, Heise et al. reported the discovery of XRFs, short transients detected by the Wide Field Cameras of BeppoSAX in the range [2-26 keV] but not seen above $40 \mathrm{keV}$ by the GRBM on-board the same spacecraft (see Boella et al. 1997, for a description of the BeppoSAX mission). In order to clarify the relationship between XRFs and GRBs, Heise et al. (2001) compared the properties (duration and spectral hardness) of 9 XRFs with the X-ray counterparts of 16 GRBs also detected in the Wide Field Cameras of BeppoSAX. They concluded that "the statistical properties of XRFs display in all aspects a natural extension of the properties of GRBs". Kippen et al. (2001) analyzed the spectra of 9 XRFs simultaneously detected by BeppoSAX/WFC and BATSE and found that XRF spectra, like those of GRBs, are well fitted by the so-called Band function consisting of two smoothly connected power laws (Band et al. 1993). Defining $x=E / E_{\mathrm{p}}$, where $E_{\mathrm{p}}$ is the peak of the $E^{2} N(E)$ spectrum we have

$N(E) \propto\left\{\begin{array}{l}x^{\alpha} \exp (-(2+\alpha) x) \quad \text { if } x \leq \frac{\alpha-\beta}{2+\alpha} \\ x^{\beta}\left(\frac{\alpha-\beta}{2+\alpha}\right)^{\alpha-\beta} \exp (\beta-\alpha) \quad \text { otherwise }\end{array}\right.$ 
$\alpha$ and $\beta$ being the two slopes respectively at low and high energy. The extended energy range of HETE-2 (from $2 \mathrm{keV}$ to $400 \mathrm{keV}$ ) has allowed to further analyze the relationship between XRFs and GRBs (Barraud et al. 2003, 2004a; Sakamoto et al. 2004; Lamb et al. 2004). It appears from these studies that XRFs are a continuation of the class of long GRBs at low energy. The distribution of their duration is indeed consistent with long GRBs. Heise et al. (2001) showed that the $t_{90}$ of 17 XRFs detected by BeppoSAX ranges from $10 \mathrm{~s}$ to $200 \mathrm{~s}$ and is comparable to the distribution of $t_{90}$ for the $36 \mathrm{GRBs}$ studied. The distribution of their spectral parameter $\alpha$ is also in agreement with the observed distribution for long GRBs. Kippen et al. (2001) showed that the distribution of $\alpha$ in the sample of XRFs and GRBs they studied is consistent with what is found for bright BATSE bursts. Moreover, Barraud et al. (2004a) found values of $\alpha$ within the range predicted by the synchrotron shock models $(-3 / 2 \leq \alpha \leq-2 / 3)$, whatever the value of $E_{\mathrm{o}}\left(E_{\mathrm{o}}\right.$ being related to the peak energy $E_{\mathrm{p}}$ by the relation $\left.E_{\mathrm{o}}=E_{\mathrm{p}} /(2+\alpha)\right)$. The distribution of the spectral parameter $\beta$ is also comparable to the distribution of $\beta$ for long bursts with a mean value of -2.5. XRFs extend the well-known hardness-intensity correlation to soft, faint bursts (Kippen et al. 2001; Barraud et al. 2003). Lamb et al. (2004) and Sakamoto et al. (2004) showed that XRFs also follow and extend the $E_{\text {iso }}-E_{\mathrm{p}}$ relation discovered by Amati et al. (2002). It is therefore now generally accepted that the XRFs, XRRs (X-Ray Rich GRBs) and classical long GRBs form a continuum, and that they share a common origin.

Using GRBs detected by BATSE, Preece et al. (2000) found a distribution of the peak energy that is narrow and centered around $200 \mathrm{keV}$. With the discovery of XRFs, Heise et al. (2001) and Kippen et al. (2001) have shown that this distribution is broader than previously thought and that it is extended towards low energies, down to a few $\mathrm{keV}$.

One of the first explanations proposed for the XRFs was that they could be GRBs observed at very high redshifts (Heise et al. 2001). This hypothesis was however discarded by Barraud et al. (2003), in view of the similar duration distributions of XRFs and long GRBs. Additionally, the first upper limits and measured spectroscopic redshifts for XRFs contradicts the high redshift hypothesis with XRF 020903 at $z=0.25$ (Soderberg et al. 2004), XRF 040701 at $z=0.215$ (Kelson et al. 2004), XRF 011030 at $z<3.5$ (Bloom et al. 2003), XRF 020427 at $z<2.3$ (Amati et al. 2004) and XRF 030723 at $z<2.1$ (Fynbo et al. 2004).

The remaining possibilities to explain XRFs are (i) GRBs with different intrinsic properties or (ii) standard GRBs viewed off-axis. In this paper we consider option (i) and study if the internal shock model of GRBs can also account for XRFs and we determine the conditions required to produce them.

\subsection{Defining an XRF}

While XRFs are best defined by their $E_{\mathrm{p}}$, the photon energy of the maximum of their $v F_{v}$ spectrum, $E_{\mathrm{p}}$ is not always available for weak soft events. Consequently, we prefer to use the ratio $R_{\mathrm{x} / \gamma}$ of the $2-30$ to the $30-400 \mathrm{keV}$ fluences to classify bursts into XRFs or GRBs. This fluence ratio is easier to compute and more robust than $E_{\mathrm{p}}$, and it has been shown that it closely reflects the value of $E_{\mathrm{p}}$ when it can be measured (Barraud et al. 2004a). Following Sakamoto et al. (2004) we consider XRFs those events with $R_{\mathrm{x} / \gamma} \geq 1$. This definition calls for the following remarks. First, the separation between XRFs and GRBs is somewhat arbitrary since the present data do not show a bimodal distribution of $E_{\mathrm{p}}$ (some authors call X-Ray Rich GRBs intermediate events with $R_{\mathrm{x} / \gamma}$ in the range 0.3 to 1 ). Second, the true fraction of XRFs depends on the definition of XRFs, but even more on the biases that affect their detection. Measuring the distances of a few XRFs could be a first step towards estimating their true fraction in a given volume. For instance, GRB 030329 (at a redshift of 0.168) would have been classified as an XRF at a redshift larger than $z=2$.

With the above definition, seven transients studied by Heise et al. (2001) and seven transients studied by Barraud et al. (2003) are XRFs. Some XRFs have been described in detail in the literature; they include XRF 020903 and XRF 030723 detected by HETE-2 (Sakamoto et al. 2004; Butler et al. 2004) and XRF 020427 detected by Beppo-SAX (Amati et al. 2004). Three events, GRB 981226 (Frontera et al. 2000), GRB 990704 (Feroci et al. 2001) and GRB 000615 (Maiorano et al. 2004) classified as X-ray rich GRBs by the SAX team are XRFs according to our definition.

\section{XRFs in the context of the internal shock model}

\subsection{A toy model for internal shocks}

The basic assumption of the internal shock scenario is that the central engine of GRBs is able to generate a relativistic wind with a highly non-uniform distribution of the Lorentz factor (with a contrast $\Gamma_{\max } / \Gamma_{\min }$ reaching at least a factor of 2 ). The observed emission is then produced when layers of different velocities collide within the wind, the dissipated energy being radiated in the gamma-ray range by means of synchrotron shock emission (Rees \& Meszaros 1994). The evolution of this relativistic wind can be followed with a hydrodynamical simulation (Daigne \& Mochkovitch 2000) but this requires large amounts of computing time which prevents one from considering a large number of cases and fully explore the parameter space. These detailed calculations have nevertheless shown that a simplified approach where the wind is represented by many shells which interact by direct collisions can also produce good results (Kobayashi et al. 1997; Daigne \& Mochkovitch 1998). The reason for this success is that the kinetic energy of the wind largely dominates over its internal energy so that pressure waves can be neglected in a first approximation. Going a step further we have developed for this study a toy model where internal shocks are limited to the collision of only two shells of equal mass $m$. Obviously, we cannot obtain from this toy model any detailed information on the temporal profiles but we expect that the main features of the burst energetics will be preserved. Shell 2 (of Lorentz factor $\Gamma_{2}$ ) is generated a time $\tau$ after shell 1 (of Lorentz factor $\Gamma_{1}<\Gamma_{2}$ ). This time interval (multiplied by $1+z$ ) represents an order of magnitude of the observed burst duration, since in the internal shock model time 
scales seen by the observer reflect the source variability. The average power injected by the central engine into the wind in this two shell approximation is given by

$\dot{E}=\frac{m c^{2}}{\tau}\left(\Gamma_{1}+\Gamma_{2}\right)=\dot{M} \bar{\Gamma} c^{2}$

where $\dot{M}=2 m / \tau$ and $\bar{\Gamma}=\left(\Gamma_{1}+\Gamma_{2}\right) / 2$ are the average mass loss rate and Lorentz factor. Shell 2 will catch up with shell 1 at the shock radius

$r_{\mathrm{s}}=2 c \tau \frac{\Gamma_{1}^{2} \Gamma_{2}^{2}}{\Gamma_{2}^{2}-\Gamma_{1}^{2}}$.

The two shells merge at $r_{\mathrm{s}}$ and the energy dissipated in the collision is given by

$E_{\text {diss }}=m c^{2}\left(\Gamma_{1}+\Gamma_{2}-2 \Gamma_{\mathrm{s}}\right)$

where $\Gamma_{\mathrm{s}}=\sqrt{\Gamma_{1} \Gamma_{2}}$ is the Lorentz factor of the shocked material in the merged shell. In order to produce a GRB this energy has to be radiated in the gamma-ray range with a characteristic broken power-law spectrum. If the synchrotron process is responsible for the emission, the peak energy (maximum of $v F_{v}$ ) is

$E_{\mathrm{p}} \sim E_{\mathrm{syn}}=C_{\mathrm{syn}} \Gamma_{\mathrm{s}} B \Gamma_{\mathrm{e}}^{2}$

where $B$ and $\Gamma_{\mathrm{e}}$ are the post shock magnetic field and electron Lorentz factor and $C_{\mathrm{syn}}=\frac{3}{4 \pi} \frac{e h}{m_{\mathrm{e}} \mathrm{c}}$. Assuming that a fraction $\alpha_{\mathrm{e}}$ of the dissipated energy is transferred to a fraction $\zeta$ of the electrons we get

$\Gamma_{\mathrm{e}}=\frac{\alpha_{\mathrm{e}}}{\zeta} \frac{m_{\mathrm{p}}}{m_{\mathrm{e}}} \epsilon$

where $\epsilon c^{2}$ is the dissipated energy per unit mass in the comoving frame.

Similarly, if a fraction $\alpha_{B}$ of the energy goes into a disordered magnetic field generated behind the shock

$B=\left(8 \pi \alpha_{B} \rho \epsilon c^{2}\right)^{1 / 2}$

the peak energy can be written as

$E_{\mathrm{p}}=C_{\mathrm{p}} \Gamma_{\mathrm{s}} \rho^{x} \epsilon^{y}$

where $\rho$ is the post shock density, $x=1 / 2, y=5 / 2$ and

$C_{\mathrm{p}}=C_{\text {syn }}\left(8 \pi \alpha_{B} c^{2}\right)^{1 / 2}\left(\frac{\alpha_{\mathrm{e}}}{\zeta} \frac{m_{\mathrm{p}}}{m_{\mathrm{e}}}\right)^{2}$.

We have considered below the possibility that $x$ and $y$ can be different from $1 / 2$ and $5 / 2$ if for example the equipartition parameters are not constant but vary with $\rho$ and/or $\epsilon$. The possibility of non-constant equipartition parameters has been considered by Chevalier (2003) and used in afterglow modelling by Yost et al. (2003) who assumed that $\alpha_{B}$ varies with the shock Lorentz factor. For the prompt phase, Daigne \& Mochkovitch (2003) have shown that the condition $2 x+y<1$ (which therefore excludes the standard values $x=1 / 2$ and $y=5 / 2)$ is often required to obtain good fits of the temporal and spectral evolution of GRB pulses.

In our two shell approximation, the physical parameters of the shocked layer $r_{\mathrm{s}}, \Gamma_{\mathrm{s}}, \rho$ and $\epsilon$ can be related to the wind

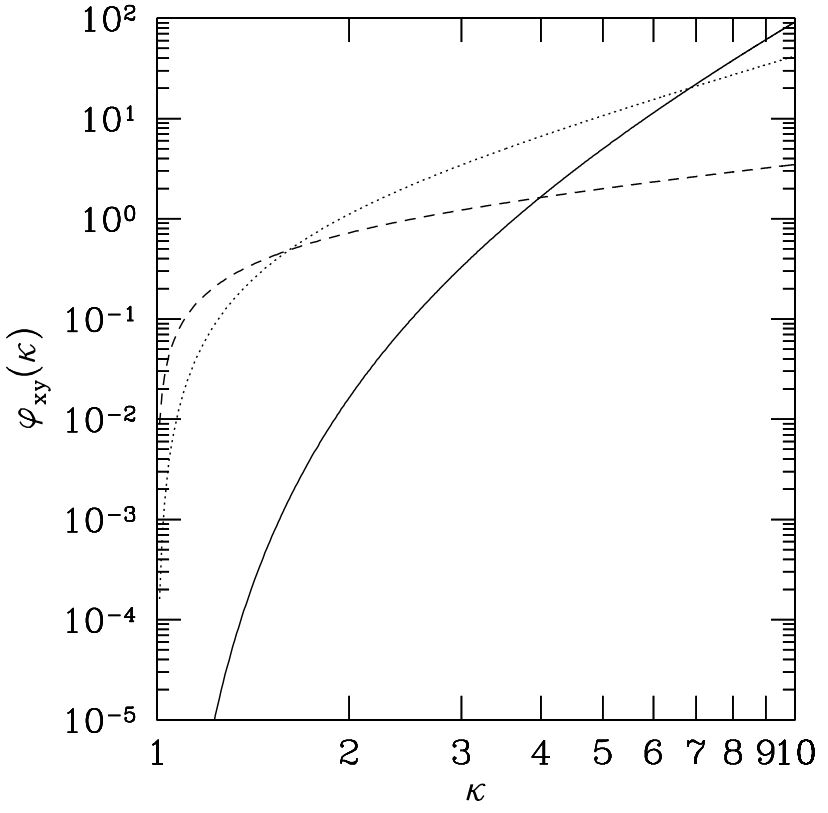

Fig. 1. The function $\varphi_{x y}(\kappa)$ for $x=1 / 2$ and $y=5 / 2$ (full line) $x=y=$ $1 / 2$ (dotted line) and $x=y=1 / 4$ (dashed line). In the first case, the large value of $y$ leads to a very steep dependence of $\varphi_{x y}$ on $\kappa$.

quantities $\dot{E}, \tau, \bar{\Gamma}$ and the contrast of Lorentz factor $\kappa=\Gamma_{2} / \Gamma_{1}$ in the following way

$r_{\mathrm{s}}=8 c \tau \bar{\Gamma}^{2} \frac{\kappa^{2}}{\left(\kappa^{2}-1\right)(\kappa+1)^{2}}$

$\Gamma_{\mathrm{s}}=\frac{2 \bar{\Gamma}}{\kappa^{1 / 2}+\kappa^{-1 / 2}}$

$\rho \sim \frac{\dot{M}}{4 \pi r_{\mathrm{S}}^{2} \bar{\Gamma} c} \sim \frac{\dot{E}}{256 \pi c^{5} \tau^{2} \bar{\Gamma}^{6}}\left(\kappa^{2}-1\right)^{2}\left(1+\frac{1}{\kappa}\right)^{4}$

$\epsilon=\frac{1}{2}\left(\kappa^{1 / 2}+\kappa^{-1 / 2}\right)-1$.

Replacing $\Gamma_{\mathrm{s}}, \rho$ and $\epsilon$ by their expressions in Eq. (8) yields

$E_{\mathrm{p}} \propto \frac{\dot{E}^{x}}{\tau^{2 x}} \frac{\varphi_{x y}(\kappa)}{\bar{\Gamma}^{6 x-1}}$

where the function

$\varphi_{x y}(\kappa)=\frac{\left[\left(\kappa^{2}-1\right)(1+1 / \kappa)^{2}\right]^{2 x}\left(\kappa^{1 / 2}+\kappa^{-1 / 2}-2\right)^{y}}{\kappa^{1 / 2}+\kappa^{-1 / 2}}$

has been represented in Fig. 1 for $\kappa=1$ to 10 and three choices of $x$ and $y$.

In spite of the simplicity of the two shell approximation, Eq. (14) predicts an anti-correlation between duration and hardness as observed in real bursts (Kouveliotou et al. 1993). Another important (and surprising) consequence of Eq. (14) is that $E_{\mathrm{p}}$ is a decreasing function of $\bar{\Gamma}$ as long as $x>1 / 6$. This can be understood from Eq. (10) which shows that internal shocks occur closer to the source in a flow with a low Lorentz factor, due to a large baryon load. If $x>1 / 6$ the reduced Lorentz factor cannot compensate for the resulting 
increase of $\rho$ (Eq. (12)) and a harder spectrum is produced. To obtain softer bursts, "clean fireballs" (i.e. with a large $\bar{\Gamma}$ ) are required. This however only applies to cases where $\bar{\Gamma}$ remains sufficiently high so that pair opacity is unimportant at the source (Meszaros \& Rees 2000). With pair creation the situation becomes more complicated and has not been considered in this paper.

\subsection{Synthetic GRBs and XRFs}

The simplicity of the two shell approximation allows us to construct large samples of synthetic bursts to check if XRFs can be produced for some specific choice of the parameters. A synthetic event is determined by the wind dynamics which is fixed by the values of $\tau, \dot{E}, \bar{\Gamma}$ and $\kappa$, the spectral parameters $C_{\mathrm{p}}, x$, $y, \alpha$ and $\beta$ and the redshift $z$. For the spectral slopes we adopt $\alpha=-1$ and $\beta=-2.5$ which correspond to the average values obtained in spectral fits of bright long GRBs performed by Preece et al. (2000). We consider three different possible choices for $x$ and $y$ : (i) $x=1 / 2, y=5 / 2$, i.e. standard equipartion assumptions; (ii) $x=y=1 / 2$, if for example the fraction of accelerated electrons is proportional to $\epsilon$ so that $\Gamma_{\mathrm{e}}$ remains approximately constant (Daigne \& Mochkovitch 1998); (iii) $x=y=1 / 4$ which was used by Daigne \& Mochkovitch (2003) in their description of the temporal and spectral evolution of GRB pulses. These smaller values of $x$ and $y$ would correspond to a situation where the dependence of the magnetic field or/and electron Lorentz factor on the dissipated energy is much weaker than in the standard case. We believe that such a possibility cannot be excluded in view of the uncertainties in the microphysics of the shocked material.

We generated a large number of events (from a few thousands to one million) by making assumptions on the distributions of the burst parameters, basing on observations or common hypothesis on the GRB physics and origin. The best constrained parameters are the redshift and the duration. If long GRBs (and XRFs) are related to the explosive death of massive stars, their rate is directly proportional to the cosmic star formation rate $\psi_{*}$ and their distribution in redshift can be deduced from $\psi_{*}(z)$ for which we have adopted the analytical expression given by Porciani \& Madau (2001) with a maximum at $z \sim 1.5$ (their SFR 1). As shown by Bloom (2003) it remains presently impossible to decide between the three possible SFRs proposed by Porciani \& Madau (2001) which can all be made compatible with the present GRB redshift data when corrected for high-redshift bias.

The distribution of the observed duration $t_{90}$ for long BATSE bursts is approximately log-normal with a maximum at $t_{90} \sim 20 \mathrm{~s}$. We have also adopted a log-normal distribution for $\tau$ with a maximum at $\tau_{\max }=10 \mathrm{~s}$ and we checked a posteriori (see Sect. 4.3) that the resulting distribution of $\tau_{\mathrm{obs}}=(1+z) \tau$ for synthetic bursts agrees with that of $t_{90}$.

The last four parameters $\bar{\Gamma}, \kappa, \dot{E}$ and $C_{\mathrm{p}}$ are much less constrained by observations and we simply take for them uniform distributions between 100 and 500 for $\bar{\Gamma}, 0$ and 1 for $\log \kappa, 51$ and 53.4 for $\log \dot{E}$ and $\log C_{\mathrm{p}}=\log C_{\mathrm{p}}^{100} \pm 0.5$ where $C_{\mathrm{p}}^{100}$ is the value of $C_{\mathrm{p}}$ which produces a typical burst

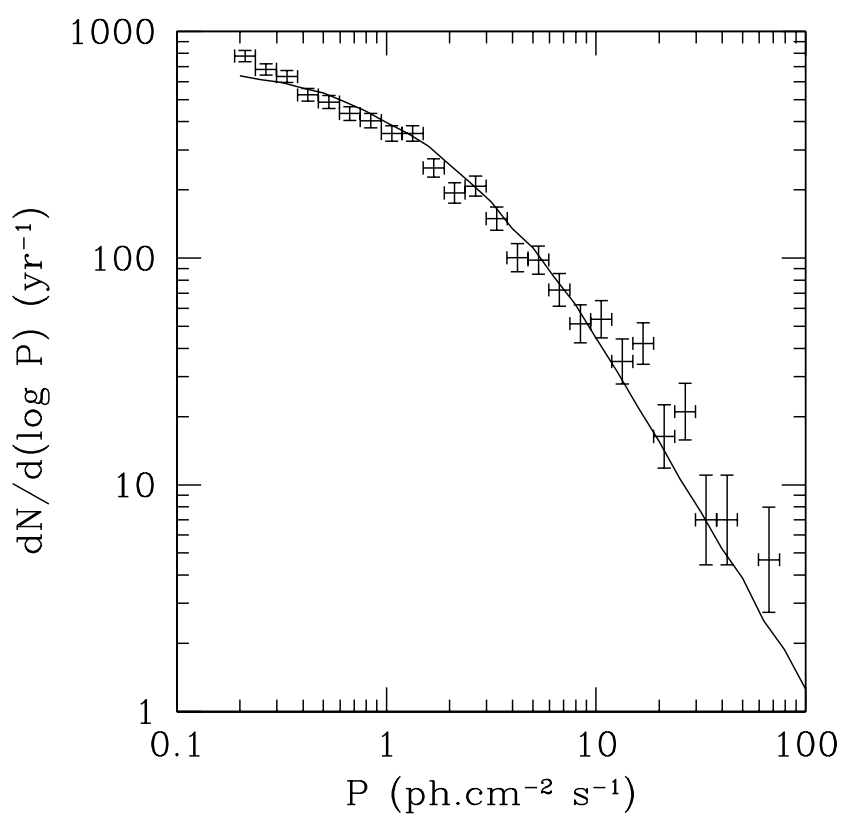

Fig. 2. Differential peak flux distribution of BATSE bursts (Stern et al. 2001) compared to a synthetic distribution $\left(10^{6}\right.$ events) with $[\log \dot{E}]_{\max }=53.4$.

with $E_{\mathrm{p}}=100 \mathrm{keV}$ if $\dot{E}=10^{52} \mathrm{erg} \mathrm{s}^{-1}, \bar{\Gamma}=300, \kappa=4, \tau=5 \mathrm{~s}$ and $z=1$. The upper limit of 53.4 for $\log \dot{E}$ has been estimated from the requirement that the synthetic $\log N-\log P$ relation agrees with the BATSE data (Stern et al. 2001). The comparison is shown in Fig. 2 for $10^{6}$ synthetic events.

The assumptions of uniform distributions for $\bar{\Gamma}, \log \kappa$, $\log \dot{E}$ and $\log C_{\mathrm{p}}$ appear to be the simplest ones considering our ignorance of the true distributions. The choice we have made also supposes that these quantities are independent, which may be wrong. Thus, we cannot expect to obtain from our results any reliable estimate of the proportion of XRFs relative to GRBs, but we can identify the range of wind paramaters that favors the production of XRFs. We will then know how the $\mathrm{XRF} / \mathrm{GRB}$ ratio varies when the distribution of these parameters differs from our initial simplest choice.

\section{Results}

\section{1. $E_{p}$ distribution and softness - fluence relation}

We first obtained the $E_{\mathrm{p}}$ distribution of our synthetic bursts. The results are shown in Fig. 3 for the three considered choices of $x$ and $y$. The full line in Fig. 3 represents the distribution for the whole sample while the dotted and dashed lines respectively correspond to the sub-groups of bursts which would have been detected by BATSE and HETE 2. A threshold of $0.2 \mathrm{ph} \mathrm{s}^{-1} \mathrm{~cm}^{-2}$ in the $50-300 \mathrm{keV}$ energy range was assumed for BATSE while for HETE 2 we adopted $1 \mathrm{ph} \mathrm{s}^{-1} \mathrm{~cm}^{-2}$ both for FREGATE (between 30 and $400 \mathrm{keV}$ ) and the WXM (between 2 and $10 \mathrm{keV}$ ). These thresholds were estimated from the work of Band (1993) for the typical energy range of each instrument. They are only indicative and in practice also depend on the burst $E_{\mathrm{p}}$ and spectral indices. This effect has not been included in our analysis. It should be rather moderate for 


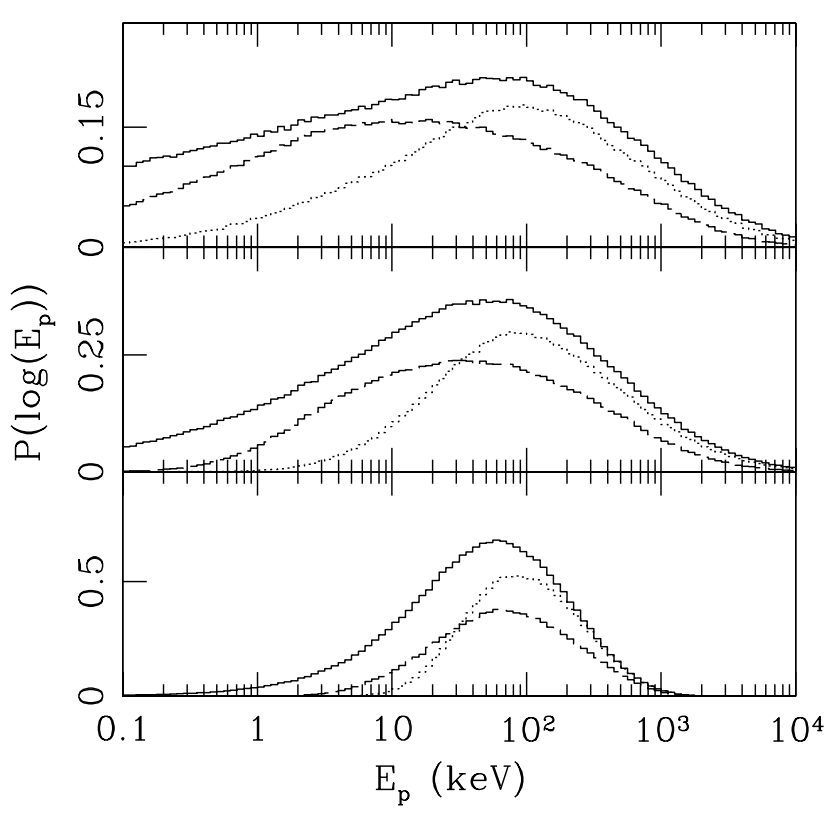

Fig. 3. Distributions of $E_{\mathrm{p}}$ for bursts that can be detected respectively by BATSE (dotted line) and by HETE 2 (dashed line). The whole sample is represented by a full line; top panel: $x=1 / 2, y=5 / 2$; middle panel: $x=y=1 / 2$; bottom panel $x=y=1 / 4$. The curves are normalized so that the integral for the whole sample $\int P\left(\log E_{\mathrm{p}}\right) \mathrm{d} \log E_{\mathrm{p}}=1$.

FREGATE and the WXM due to the relative flatness of the sensitivity curves (Band 1993). In the case of BATSE it will contribute to decrease the already small number of detected events at low energy.

For $x=1 / 2$ and $y=5 / 2$ and $x=y=1 / 2$ the distribution of $E_{\mathrm{p}}$ for BATSE bursts is wider than the observed one, which is confined between $10 \mathrm{keV}$ and $1 \mathrm{MeV}$ (Preece et al. 2000). Conversely, the agreement is excellent for $x=y=1 / 4$ (since the value of $E_{\mathrm{p}}$ is then much less sensitive to the dispersion of the wind parameters). We have therefore adopted $x=y=1 / 4$ in the remainder of this paper since it appears that this choice of $x$ and $y$ gives the best results both for individual bursts (Daigne \& Mochkovitch 2003) and statistically for a large population.

Figure 3 shows that BATSE misses most of the low $E_{\mathrm{p}}$ events while HETE 2, which is less sensitive than BATSE in hard X-rays but has a lower energy threshold, can detect at least part of them down to $E_{\mathrm{p}} \sim$ a few $\mathrm{keV}$ as was the case for Beppo-SAX. This is also illustrated in Fig. 4 where we have represented the softness $R_{\mathrm{x} / \gamma}$ as a function of the total (2-400 keV) fluence for a population of 1450 synthetic events which would have been detected by HETE 2 . The total number of events produced was 3000 , so that the detection fraction was about $1 / 2$ (a smaller number of bursts was used in this case to avoid confusion in the figure). The two limits of the softness at 0.075 and 4 respectively correspond to the hardest and softest bursts for which the two bands $2-30$ and $30-400 \mathrm{keV}$ are both in the low or high energy part of the spectrum. With the

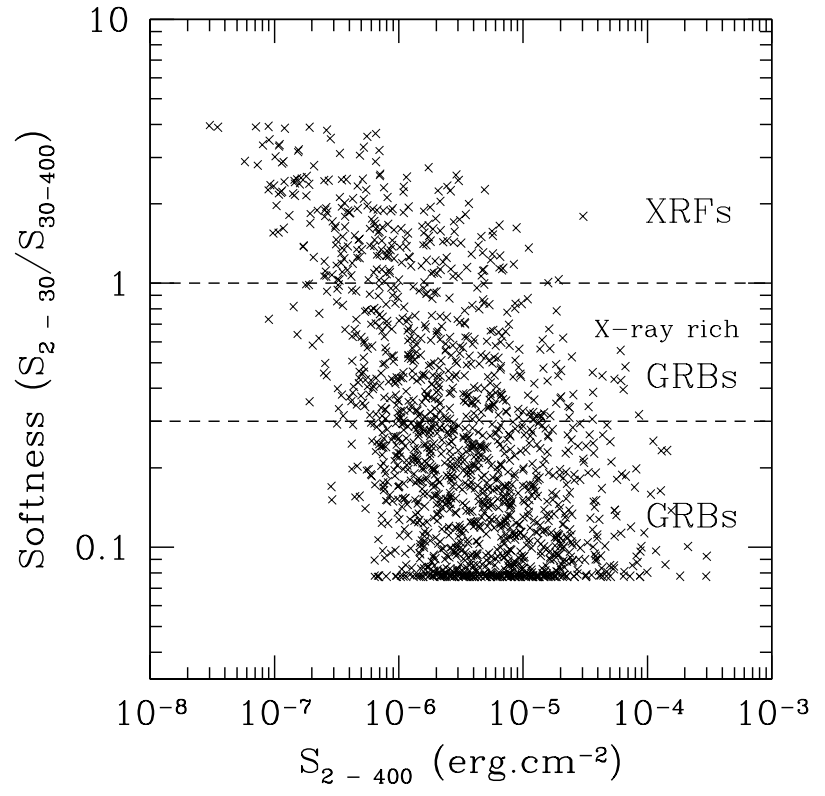

Fig. 4. Softness versus total fluence for a population of 1450 synthetic events that can be detected by HETE 2 .

assumed values of $\alpha=-1$ and $\beta=-2.5$ the softness limits are simply given by

$R_{\mathrm{x} / \gamma}=\frac{\int_{2}^{30} \mathrm{~d} E}{\int_{30}^{400} \mathrm{~d} E}=0.0757$

for hard events and

$R_{\mathrm{x} / \gamma}=\frac{\int_{2}^{30} E^{-1.5} \mathrm{~d} E}{\int_{30}^{400} E^{-1.5} \mathrm{~d} E}=3.957$

for soft events. The two horizontal dashed lines in Fig. 4 separate the GRB, X-ray rich GRB and XRF domains. In agreement with Fig. 3 it can be seen that the model generates a population of XRFs that can be detected by HETE 2 (but would have mostly escaped detection by BATSE). Since, from the toy model, we know all the input parameters of these synthetic $\mathrm{XRFs}$, we can identify the key ingredients necessary to produce them. For this purpose we compare below the distributions of $z$, $\tau, \dot{E}, \bar{\Gamma}$ and $\kappa$ between XRFs and standard GRBs.

\subsection{Redshift distribution}

Figure 5 shows that the redshift distributions of synthetic GRBs (dotted line) and XRFs (full line) are very similar (the adopted normalization is $\left.\int P(\log (1+z)) \operatorname{dlog}(1+z)=1\right)$. In the context of our simulation, XRFs are not standard GRBs observed at large $z$. This was already strongly suggested by (i) the duration distribution of the observed XRFs which is comparable to that of long GRBs and (ii) the recent redshift determinations (or upper limits) obtained for several XRFs (see Sect. 2.1). Nevertheless, even at large redshift a bright GRB is still observable (Lamb \& Reichart 2000) but can appear as an XRF so that the XRF/GRB ratio is expected to increase with $z$. Figure 5 shows that this is indeed the case: the $\mathrm{XRF} / \mathrm{GRB}$ ratio at $z>5$ 


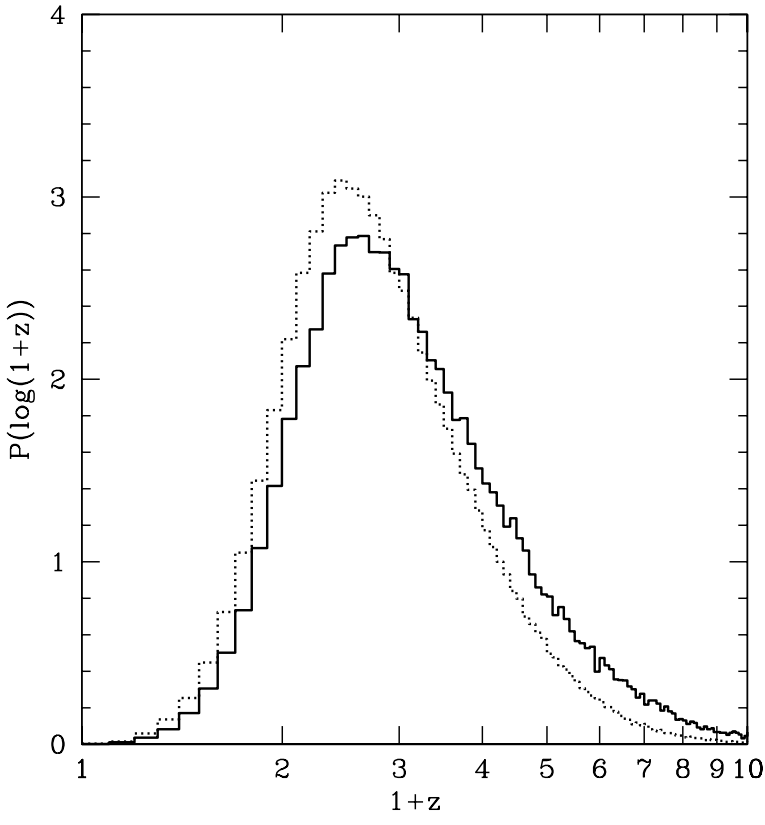

Fig. 5. Redshift distribution of synthetic GRBs (dotted line) and XRFs (full line) obtained with our toy model.

is more than 2 times larger than at $z=1$. However, the number of events at large $z$ is not sufficient to account for the bulk of the XRF population. We have checked that this was not a consequence of our specific choice for $\psi_{*}(z)$ which is maximum at $z=1.5$. With SFR 2 of Porciani \& Madau (2001) which is nearly constant at $z>2$, the distributions of both GRBs and XRFs remain similar, being only slightly shifted to larger $z$. In any case, most events stay confined between $z=1$ and 5 and the majority of XRFs are not GRBs at large $z$.

Our synthetic XRFs are therefore intrinsically soft due to some specific values of their relativistic wind parameters.

\subsection{Distribution of the wind parameters in GRBs and XRFs}

We compare in Fig. 6 the distribution of the four wind parameters $(\tau, \bar{\Gamma}, \kappa, \dot{E})$ in synthetic GRBs and XRFs.

Observed duration: the distribution of the observed duration $\tau(1+z)$ in GRBs and XRFs is represented in Fig. 6a. As expected, it is in good agreement with the BATSE duration distribution for long GRBs. The average duration of XRFs is approximately $50 \%$ longer. This is not a consequence of a larger redshift but of a preferred longer intrinsic duration resulting from the duration-hardness relation $E_{\mathrm{p}} \propto \tau^{-1 / 2}$ for $x=1 / 4$ (Eq. (14)).

Average Lorentz factor: the average Lorentz factor in GRBs closely follows the uniform input distribution while large values of $\bar{\Gamma}$ are favored in XRFs. This is again a consequence of Eq.14 since $E_{\mathrm{p}} \propto \bar{\Gamma}^{-1 / 2}$ for $x=1 / 4$.

Contrast of the Lorentz factor: the distribution of the contrast $\kappa$ shows a striking difference between GRBs and XRFs. XRFs appears to be produced by relativistic winds where the contrast typically does not exceed a factor of 4 . The maximum of the
XRF distribution is located at $\kappa=1.4$. Conversely, the proportion of GRBs steadily increases with $\kappa$. As a consequence of the small contrast of $\Gamma$ in XRFs the efficiency for energy dissipation by internal shocks

$f=\frac{E_{\mathrm{diss}}}{m c^{2}\left(\Gamma_{1}+\Gamma_{2}\right)}=\frac{\kappa^{1 / 2}+\kappa^{-1 / 2}-2}{\kappa^{1 / 2}+\kappa^{-1 / 2}}$

is small, close to $1 \%$ at the maximum of the contrast distribution.

Injected power: the distribution of the injected power $\dot{E}$ shows that large $\dot{E}$ are favored in both GRBs and XRFs because events with low injected power often escape detection. XRFs are therefore not characterized by a deficit of injected power (even if the largest $\dot{E}$ are more frequently found in GRBs). They appear weak and soft due to the inefficiency of their internal shocks. The dissipated energy in XRFs and GRBs is compared in Fig. 7. The two distributions peak at $7 \times 10^{51}$ and $6 \times$ $10^{52}$ erg respectively. Again, this difference of nearly a factor of ten comes from the lower efficiency of internal shocks in XRFs relative to GRBs. Finally, the shock parameters, through the value of $C_{\mathrm{p}}$, also show some differences between GRBs and XRFs. A smaller $C_{\mathrm{p}}$ naturally favors the production of an XRF but a reduction of $C_{\mathrm{p}}$ alone is not enough since, contrary to a low $\kappa$, it increases the softness without simultaneously decreasing the radiated power (Barraud et al. 2004b).

\subsection{Amati relation}

Assuming that a constant fraction $\alpha_{\mathrm{e}}$ of the dissipated energy is transferred to the electrons and radiated, it is possible to check if our synthetic bursts follow the Amati relation between the isotropic radiated energy and the value of $E_{\mathrm{p}}$ in the burst rest frame (Amati et al. 2002). The results are shown in Fig. 8 for $\alpha_{\mathrm{e}}=0.3$, this rather large value of $\alpha_{\mathrm{e}}$ being required to maintain a reasonable overall efficiency

$f=\alpha_{\mathrm{e}} \times f_{\mathrm{IS}}$

where $f_{\mathrm{IS}}$ is the efficiency of dissipation by internal shocks. Bursts which could be detected by HETE 2 have been represented by large dots in Fig. 8. For this sub-group, the best fit by a power law gives

$E_{\mathrm{p}}=200\left(\frac{E_{\mathrm{rad}}}{10^{52} \mathrm{erg}}\right)^{0.46} \mathrm{keV}$.

The exponent is close to 0.5 as found in the observational Amati relation, which now extends over five orders of magnitude in $E_{\text {rad }}$ when XRF 020903 is included (Sakamoto et al. 2004). We however notice that the dispersion of synthetic bursts relative to the power law is larger than for observed bursts. If the small dispersion of the observational Amati relation is confirmed in the future with a larger number of bursts it will provide a strong constraint, probably indicating that a new physical ingredient - such as a correlation between some of the wind parameters - should be included in the models. 

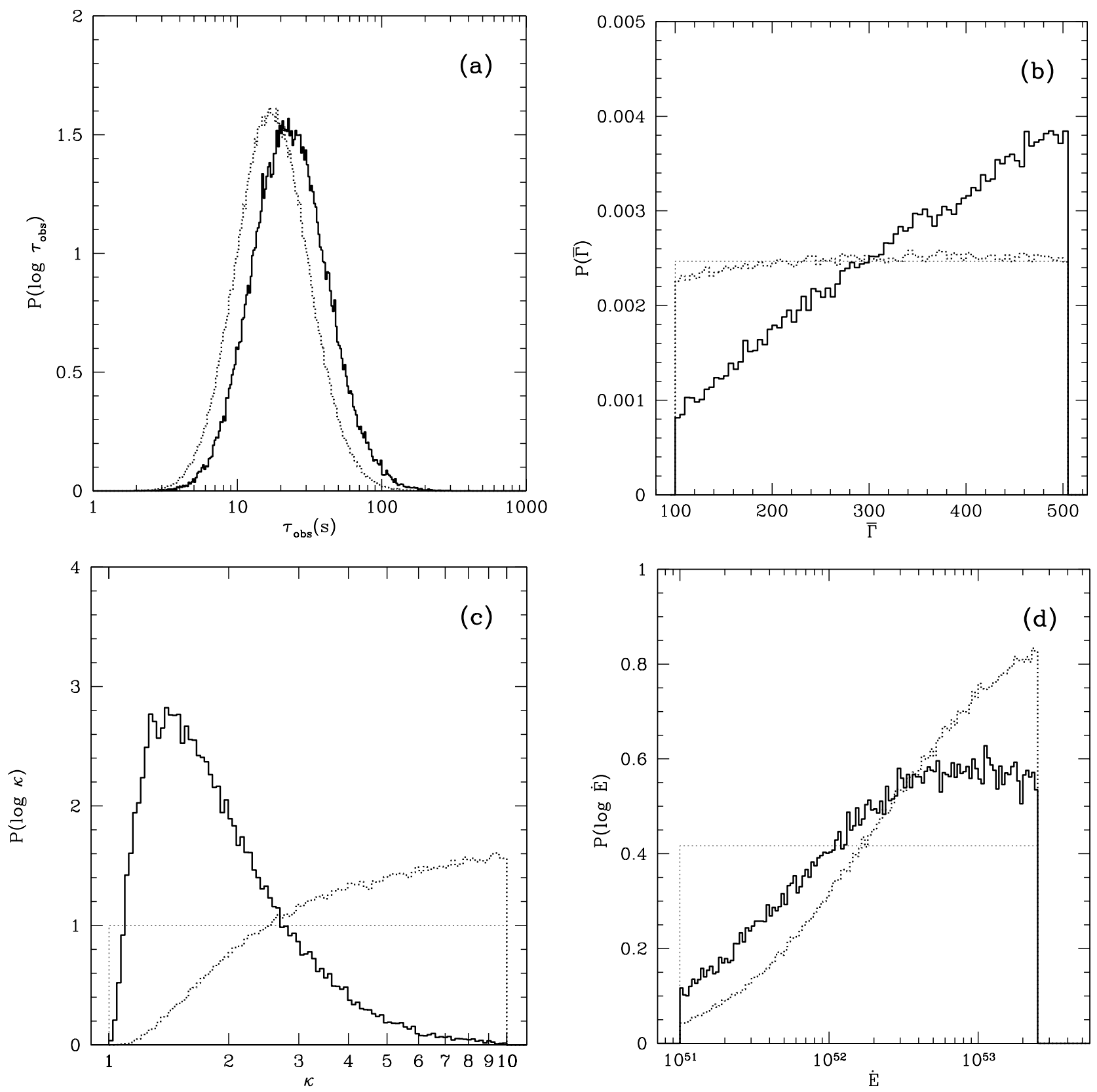

Fig. 6. Distributions of $\left.\left.\left.\tau_{\mathrm{obs}}=(1+z) \tau \mathbf{a}\right), \bar{\Gamma} \mathbf{b}\right), \log \kappa \mathbf{c}\right)$ and $\left.\log \dot{E} \mathbf{d}\right)$ for GRBs (dotted line) and XRFs (full line). The thin dotted lines in (b)-d)) represent the uniform distributions used as input. The adopted normalizations are $\int P\left(\log \tau_{\mathrm{obs}}\right) \mathrm{d} \log \tau_{\mathrm{obs}}=1, \int_{100}^{500} P(\bar{\Gamma}) \mathrm{d} \bar{\Gamma}=1$, $\int_{0}^{1} P(\log \kappa) \mathrm{d} \log \kappa=1$ and $\int_{51}^{53.4} P(\log \dot{E}) \mathrm{d} \log \dot{E}=1$.

\section{Conclusion}

We have used a simple internal shock model to generate a large number of GRBs with different relativistic wind parameters such as the average Lorentz factor $\bar{\Gamma}$, the contrast $\kappa$ between the maximum and minimum Lorentz factor or the injected power $\dot{E}$. We adopted a lognormal distribution of the intrinsic duration and obtained the redshift distribution assuming that the burst rate is proportional to the cosmic star formation rate. We also assumed standard values for the low and high energy slopes of the spectrum, $\alpha=-1$ and $\beta=-2.5$ and discussed different possibilities regarding the shock parameters. Our aim was to identify the physical conditions leading to the formation of XRFs. We have found that our synthetic XRFs exhibit distributions of redshift, duration and injected power rather similar to those of GRBs but strongly differ in the distributions of $\bar{\Gamma}$ and $\kappa$. XRFs are events where the contrast of Lorentz factors is small, predominantly between 1 and 2 .

Since we do not know the true distributions of these parameters we cannot make any prediction on the relative fraction of XRFs and GRBs. With the uniform distributions adopted for $\bar{\Gamma}$ and $\log \kappa$ we obtain $16 \%$ of XRFs, $27 \%$ of X-ray rich GRBs and $57 \%$ of GRBs. This is not in agreement with the HETE results which show approximately equal fractions of the 


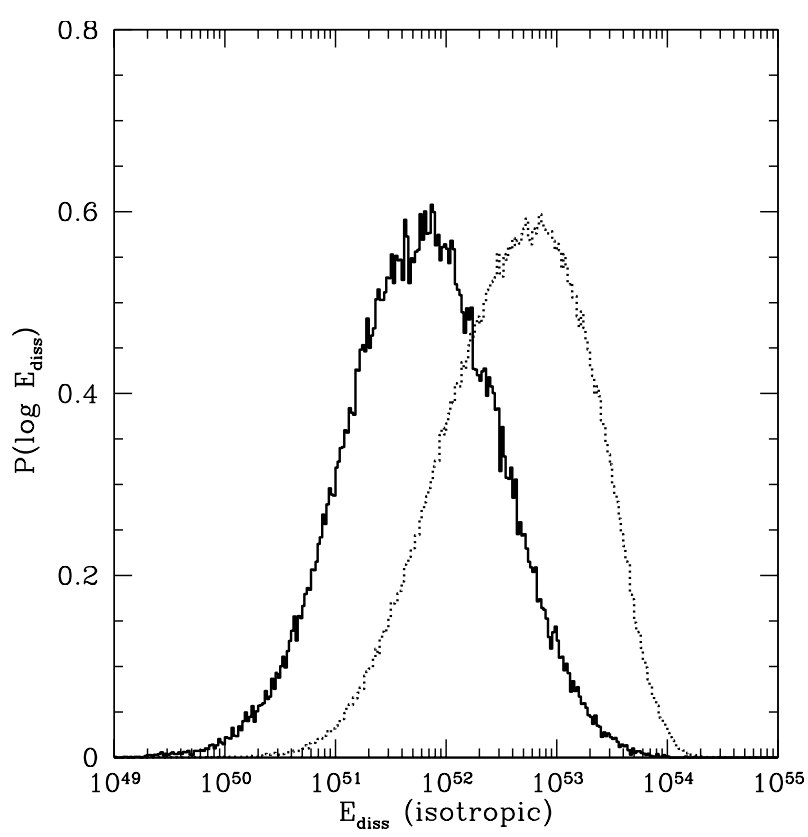

Fig. 7. Distribution of the dissipated energy in synthetic GRBs (dotted line) and XRFs (full line).

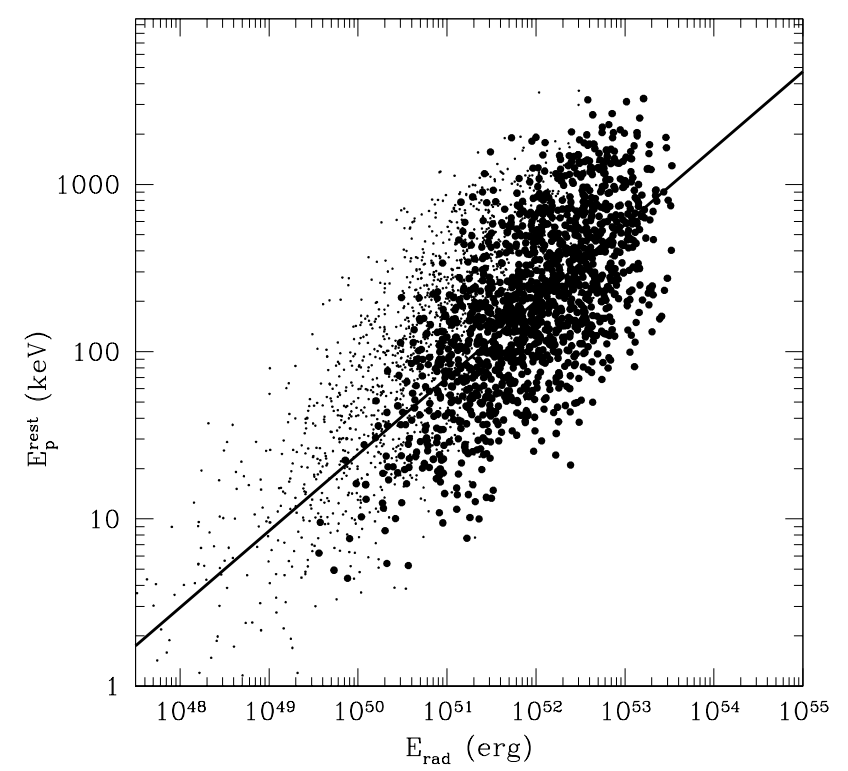

Fig. 8. The Amati relation for synthetic burts. A sample of 3000 synthetic events is represented, the large dots corresponding to those which can be detected by HETE 2 . The line is the best fit for the HETE sub-sample.

three classes (Barraud et al. 2004a). If however the weight of events with a low contrast of Lorentz factors is increased, for example with a distribution of $\log \kappa$ which remains uniform but extends to 0.6 only, so that the maximum contrast is 4 instead of 10 , the agreement with the HETE results is improved with now $31 \%$ of XRFs, $33 \%$ of X-ray rich GRBs and $36 \%$ of GRBs.

If XRFs are produced by internal shocks with a low contrast in the Lorentz factor distribution, the efficiency of energy dissipation is expected to be smaller than in GRBs. This can in principle be tested from multiwavelength fits of the afterglow lightcurves and spectra. Such a study including for the first time an XRF (XRF 020903) was recently presented by Lloyd-Ronning \& Zhang (2004). The efficiency for XRF 020903 indeed appears to be much smaller than what is found for the GRBs of the sample. However all the efficiencies measured by Lloyd-Ronning \& Zhang (2004) are quite large (0.1 for XRF 020903, between 0.4 and 1 for the GRBs) which can be very challenging for internal shock models.

If conversely the Lorentz factor is always highly variable so that small values of $\kappa$ rarely occur one should then look for another origin for XRFs such as viewing angle effects. This possibility has been studied in detail by Yamazaki et al. (2002, 2004). It supposes that the jets responsible for GRBs are uniform and have sharp edges. If these assumptions are verified, Yamazaki et al. $(2002,2004)$ have shown that many of the observed and statistical properties of XRFs can be accounted for by assuming a distribution of the jet opening angle such as $\Delta \theta \propto \Delta \theta^{-2}$ (with $10^{-2}<\Delta \theta<10^{-1} \mathrm{rad}$ ), XRFs being then obtained for large viewing angles $\theta_{v}>\Delta \theta$.

Of course, the intrinsic and extrinsic origins for the softness of XRFs do not exclude each other. A low $E_{\mathrm{p}}$ can result from both a small contrast and a large viewing angle. Their respective contributions to the XRF population could be evaluated, for different distributions of $\kappa$ and $\Delta \theta$, by adding the viewing angle effect to the present study.

Acknowledgements. The authors would like to thank the anonymous referee for an extremely careful reading of the manuscript. His/her comments and remarks have strongly contributed improving our paper.

\section{References}

Amati, L., Frontera, F., Tavani, M., et al. 2002, A\&A, 390, 81

Amati, L., Frontera, F., in't Zand, J. J. M., et al. 2004, A\&A, 426, 415 Band, D. 2003, ApJ, 588, 945

Band, D., Matteson, J., Ford, L., et al. 1993, ApJ, 413, 281

Barraud, C., Olive, J.-F., Lestrade, J. P., et al. 2003, A\&A, 400, 1021

Barraud, C., and the HETE-2 Team 2004a, Proc. of the 2003 GRB Conf. held in Santa Fe, NM, USA, Sept. 8-12, 2003

Barraud, C., Daigne, F., Mochkovitch, R., \& Atteia, J. L. 2004b, Proc. 2003 GRB Conf. held in Santa Fe, NM, USA, Sept. 8-12, 2003

Bloom, J. S. 2003, AJ, 125, 2865

Bloom, J. S., Fox, D., van Dokkum, P. G., et al. 2003, ApJ, 599, 957

Boella, G., Butler, R., Perola, G., et al. 1997, A\&AS, 122, 299

Butler, N., Dullighan, A., Ford, P., et al. 2004 [arXiv: astro-ph/0401020]

Chevalier, R. A. 2003, Supernovae: 10 Years of 1993J, ed. J. M. Marcaide, \& K. W. Weiler (Springer Verlag), IAU Colloq., 192

Daigne, F., \& Mochkovitch, R. 1998, MNRAS, 296, 275

Daigne, F., \& Mochkovitch, R. 2000, A\&A, 358, 1157

Daigne, F., \& Mochkovitch, R. 2003, MNRAS, 342, 587

Feroci, M., Antonelli, L. A., Soffita, P., et al. 2001, A\&A, 378, 441

Fynbo, J. P. U., Sollerman, J., Hjorth, J., et al. 2004, ApJ, 609, 962

Frontera, F., Antonelli, L. A., Amati, L., et al. 2000, ApJ, 540, 697 
Heise, J., in't Zand, J., Kippen, R. M., \& Woods, P. M. 2001, Gamma-Ray Bursts in the Afterglow Era, ed. Enrico Costa, Filippo Frontera, \& Jens Hjorth (Berlin Heidelberg: Springer), 16 Kelson, D. D., Koviak, K., Berger, E., \& Fox, D. B. 2004, GCN, 2627 Kippen, R. M., Woods, P. M., Heise, J., et al. 2001, Gamma-Ray Bursts in the Afterglow Era, ed. Enrico Costa, Filippo Frontera, \& Jens Hjorth (Berlin Heidelberg: Springer), 22

Kobayashi, S., Piran, T., \& Sari, R. 1997, ApJ, 490, 92

Kouveliotou, C., Meegam, C. A., Fishman, G. J., et al. 1993, ApJ, 413, L101

Lamb, D. Q., \& Reichart, D. E. 2000, ApJ, 536, 1

Lamb, D. Q., Donaghy, T. Q., \& Graziani, C. 2004, New Astr. Rev., 48,459

Lloyd-Ronning, N. M., \& Zhang, B. 2004, ApJ, 601, 371

Maiorano, E., Masetti, N., Palazzi, E., et al. 2004, Third Rome Workshop on Gamma-Ray Bursts in the Afterglow Era, ed. Marco Feroci, Filippo Frontera, Nicola Masetti, \& Luigi Piro, ASP Conf. Ser., 312, 221
Meszaros, P., \& Rees, M. J. 2000, ApJ, 530, 292

Porciani, C., \& Madau, P. 2001, ApJ, 548, 522

Preece, R. D., Briggs, M. S., Mallozi, R. S., et al. 2000, ApJS, 126, 19 Rees, M. J., \& Meszaros, P. 1994, ApJ, 430, L93

Ricker, G., Atteia, J.-L., Crew, G., et al. 2001, AIP Conf. Proc., 662, 3 Sakamoto, T., Lamb, D. Q., Graziani, C., et al. 2004, ApJ, 602, 875

Soderberg, A. M., Kulkarni, S. R., Berger, E., et al. 2004, ApJ, 606, 994

Stern, B. E., Tikhomirova, Y., Kompaneets, D., Svensson, R., \& Poutanen, J. 2001, ApJ, 563, 80

Yamazaki, R., Ioka, K., \& Nakamura, T. 2002, ApJ, 571, L31

Yamazaki, R., Ioka, K., \& Nakamura, T. 2004, ApJ, 607, L103

Yost, S. A., Harrison, F. A., Sari, R., \& Frail, D. A. 2003, ApJ, 597, 459

Zhang, B., \& Meszaros, P. 2002, ApJ, 581, 1236

Zhang, B., Dai, X., Lloyd-Ronning, N. M., \& Meszaros, P. 2004, ApJ, 601, L119 